\title{
Combining sequence-specific probes and DNA binding dyes in real-time PCR for specific nucleic acid quantification and melting curve analysis
}

\author{
Kristina Lind ${ }^{1,2}$, Anders Ståhlberg ${ }^{2,3}$, Neven Zoric ${ }^{2}$, and Mikael Kubista ${ }^{1,2}$ \\ ${ }^{1}$ Chalmers University of Technology, Gothenburg, ${ }^{2}$ TATAA Biocenter, Gothenburg, \\ and ${ }^{3}$ Lund University, Lund, Sweden \\ BioTechniques 40:315-319 (March 2006) \\ doi 10.2144/000112101
}

Currently, in real-time PCR, one often has to choose between using a sequence-specific probe and a nonspecific double-stranded DNA (dsDNA) binding dye for the detection of amplified DNA products. The sequence-specific probe has the advantage that it only detects the targeted product, while the nonspecific dye has the advantage that melting curve analysis can be performed after completed amplification, which reveals what kind of products have been formed. Here we present a new strategy based on combining a sequence-specific probe and a nonspecific dye, BOXTO, in the same reaction, to take the advantage of both chemistries. We show that BOXTO can be used together with both TaqMan ${ }^{\circledR}$ probes and locked nucleic acid (LNA) probes without interfering with the PCR. The probe signal reflect formation of target product, while melting curve analysis of the BOXTO signal reveals primer-dimer formation and the presence of any other anomalous products.

\section{INTRODUCTION}

During recent years, the use of realtime PCR to quantify nucleic acids has grown extensively. It is currently the most sensitive method to determine the amount of specific DNA sequences in complex biological samples and has been applied in such different areas as cancer diagnostics $(1,2)$, food and veterinary diagnostics $(3,4)$, and basic genomic research (5). Detection can be performed by using sequence-specific probes such as TaqMan ${ }^{\circledR}$ probes (6), molecular beacons (7), hybridization probes (8), locked nucleic acid (LNA) probes (www.biotechniques. com/article.asp?id=91200423), and LightUp ${ }^{\circledR}$ probes (9). Alternatively, free dyes that bind to double-stranded DNA (dsDNA) such as SYBR ${ }^{\circledR}$ Green I (10) and BEBO (11) can be used. When using sequence-specific probes, only the targeted product is detected, while all dsDNA contributes to the fluorescence signal of DNA binding dyes. In addition to the correct PCR product, this includes primer-dimers and other nonspecific amplification products. Primer-dimer formation is a major problem when quantifying low amounts of DNA due to competition between the two amplification reactions, which often results in a reduced sensitivity of the assay. DNA binding dyes reflect formation of both the correct product and the primerdimers and may overestimate the target DNA concentration. When sequencespecific probes are used, primer-dimer products are not seen, but they may still form and interfere with the amplification reaction of the targeted product. An advantage of DNA binding dyes is that melting curve analysis can be performed after the amplification in a closed-tube format to identify the PCR products that have been formed (12). This is not possible when only using sequence-specific probes. In melting curve analysis, the temperature is gradually increased while monitoring the fluorescence. When the temperature is reached that melts the dsDNA, the dye is released, and its fluorescence decreases. The melting temperature depends on the sequence of the DNA, but most importantly, on the length of the dsDNA product. Hence, different PCR products may be distinguished by melting curve analysis.

The most common fluorophore used in probes is FAM, which is a derivative of fluorescein (13). FAM has an excitation maximum at $493 \mathrm{~nm}$ and an emission maximum at $525 \mathrm{~nm}$. SYBR Green I, which is the most popular DNA binding dye for real-time PCR, has almost identical maxima, 497 and $521 \mathrm{~nm}$, respectively, and the FAM and SYBR Green I spectra overlap extensively. Their responses are measured in the same detection channel or using the same filter settings in the real-time PCR instruments (14), making it impossible to distinguish the signals from these dyes. A new dsDNA binding dye, BOXTO, was recently developed (15). BOXTO is an asymmetrical cyanine dye that is similar to SYBR Green I (16), but with an excitation maximum at $515 \mathrm{~nm}$ and an emission maximum at $552 \mathrm{~nm}$. These spectral properties are distinct from FAM, and BOXTO can be used in the same reaction as FAM without interfering spectroscopically with it by using appropriate detection channels or filters.

In this work, we demonstrate the novel strategy of combining a nonspecific dsDNA binding dye (BOXTO) with either a TaqMan or an LNA probe in the same reaction. The sequencespecific probe is used to quantify target DNA molecules, while the nonspecific BOXTO is used for melting curve analysis to reveal formation of nonspecific PCR products. The whole process is performed in a homogenous solution in closed reaction containers. The approach is demonstrated on three PCR systems with varying amounts of primer-dimers and erroneous PCR products. One assay was designed for the amyloid $\beta$ (A4) precursor protein gene (amyloid assay), one for the Escherichia coli $\beta$-glucoronidase gene (gusA assay), and one for the nanog gene (nanog assay).

\section{MATERIALS AND METHODS}

\section{Primers, Probes, and Template}

Primer sequences for the amyloid assay were 5'-ATGGGTTGGCCGCT- 
Figure 1. Standard curves, gene expression profiling, and melting curves using sequence-specific probes in combination with BOXTO. Standard curves were constructed from experiments with only probe (triangles) and experiments with probe and BOXTO in the same reaction tube [cycle threshold $\left(\mathrm{C}_{\mathrm{T}}\right)$ values from the probe channel are shown as circles and those from the BOXTO channel as squares]. Standard curves of (A) TaqMan-probed amyloid assay and (B) locked nucleic acid (LNA)probed gusA assay are shown. The part of the standard curves where samples contain mostly the targeted PCR product are shown with solid line in blue, while the part of the standard curve suffering from interference by primer-dimer formation is shown with dashed lines in red. Samples with $C_{T}$ values of 40 or higher were considered negative. These are shown in the top of the graphs and were not considered when drawing the standard curves. The amyloid and gusA PCR assays were run with regular (not hot start) Taq DNA polymerase, and they produced significant amounts of primer-dimers. The insets show melting curves of three selected samples from the reaction monitored with both probe and BOXTO. They were selected to contain targeted PCR product only (blue), a mixture of targeted PCR product and primerdimers (black), and primer-dimers only (red). Note that samples of the standard curves marked red in panels A and B contain both samples with primer-dimers only (red melting curve) and samples with a mixture of target PCR product and primer-dimers (black melting curve). (C) The expression of nanog measured by the probe signal is shown. Positive samples are shown in blue. The nanog expression decreases with time as reflected by increasing $C_{T}$ values. Melting curve analysis based on the BOXTO signal revealed formation of an aberrant PCR product, with a higher melting temperature (approximately $90^{\circ} \mathrm{C}$ ) than the targeted product (approximately $87^{\circ} \mathrm{C}$ ), in the biological negative control (green melting curve). This RNA was extracted from mouse. The no template control (NTC) samples, shown in red, contained only primer-dimers. The melting temperatures of the full-length PCR products were significantly higher than that of the corresponding primer-dimers (approximately $80^{\circ} \mathrm{C}$ ) for all three assays studied. hESC, human embryonic stem cells.
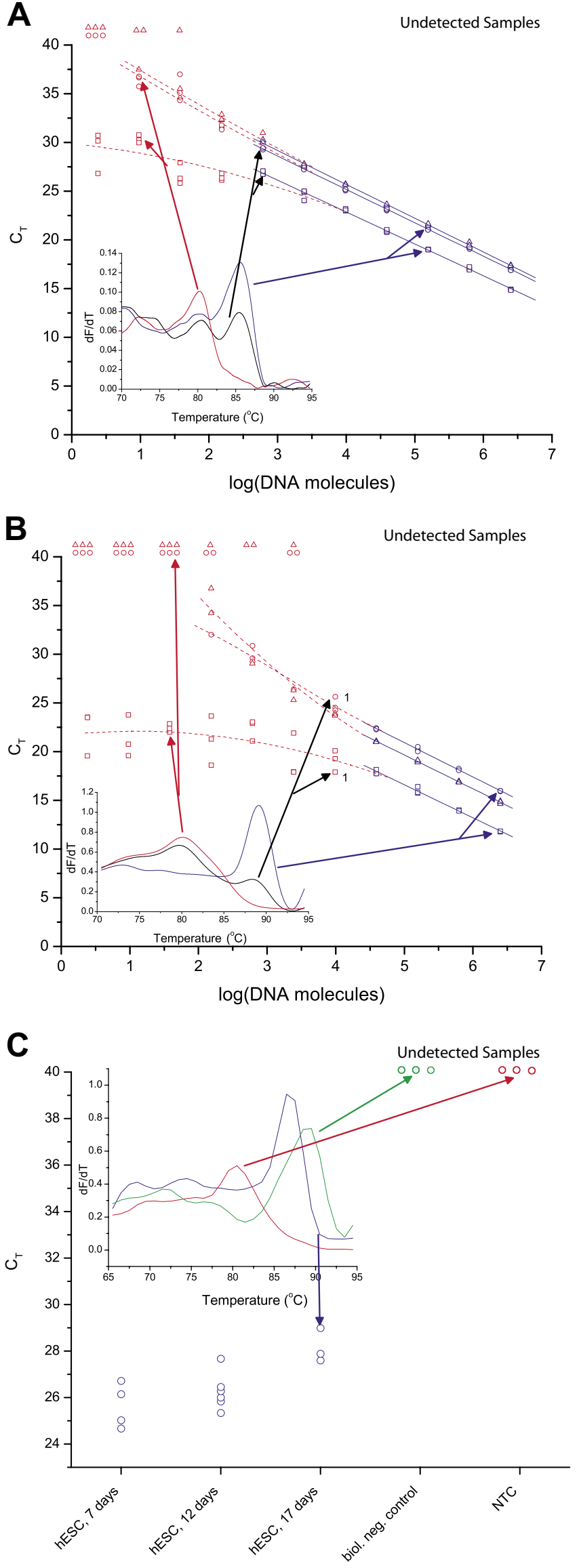

TCTTTG- $3^{\prime}$ and $5^{\prime}$-GCTTGTTGAGACAGCAAAACCTC-3', which produce a 133-bp product. The TaqMan probe sequence was 5'-CCTGCGCCTTGCTCCTTTGGTTCG-3' and was labeled with FAM at the $5^{\prime}$ end and Black Hole Quencher ${ }^{\mathrm{TM}} 1$ (Biosearch Techniologies, Novato CA, USA) at the $3^{\prime}$ end. Primer sequences for the gusA and nanog assays were 5'-CTCTTTGATGTGCTGTGCCT-3' (gusA forward) and 5'-ACATATCCAGCCATGCACAC-3' (gusA reverse), which produce a 223 -bp product. The nanog assay was run as previously described (17). Sequence matching LNA probes (number 51 for gusA and number 69 for nanog) from the Human ProbeLibrary $^{\mathrm{TM}}$ (Exiqon Vedbaek, Denmark) were used. The probes were labeled with fluorescein and a nonfluorescent quencher according to the manufacturer. All measurements for amyloid and gusA were performed using purified PCR products as a template, while cDNA from human stem cells was used for the nanog assay, as described elsewhere (17).

\section{Real-Time PCR Conditions}

Real-time PCRs for the amyloid system contained $75 \mathrm{mM}$ Tris, $\mathrm{pH} 8.8,20 \mathrm{mM}$ $\left(\mathrm{NH}_{4}\right)_{2} \mathrm{SO}_{4}, 0.1 \%$ Tween ${ }^{\circledR} 20$, $4 \mathrm{mM} \mathrm{MgCl}_{2}$ (Sigma-Aldrich, Stockholm, Sweden), $0.2 \mathrm{mM}$ of each dNTP (Sigma-Aldrich), $0.3 \mu \mathrm{M}$ of each primer (MWGBiotech, Ebersberg, Germany), 1 U Taq DNA polymerase (SigmaAldrich), $0.2 \mu \mathrm{M}$ TaqMan probe (Eurogentec, Liege, Belgium), and/or $0.3 \mu \mathrm{M}$ BOXTO (TATAA Biocenter, Gothenburg, Sweden). For the gusA assay, the reactions contained $10 \mathrm{mM}$ Tris- $\mathrm{HCl}, \mathrm{pH}$ 8.3, and $50 \mathrm{mM} \mathrm{KCl}, 3.5 \mathrm{mM}$ $\mathrm{MgCl}_{2}, 0.2 \mathrm{mM}$ of each dNTP, $0.3 \mu \mathrm{M}$ of each primer, $1 \mathrm{U} \mathrm{Taq}$ DNA polymerase, $0.1 \mu \mathrm{M}$ LNA probe, and/or $0.6 \mu \mathrm{M}$ BOXTO. For the nanog assay, the reactions contained $10 \mathrm{mM}$ Tris- $\mathrm{HCl}, \mathrm{pH}$ 
Table 1. Detection Chemistry Combinations and PCR Efficiencies

\begin{tabular}{|llcc|}
\hline Target & Detection Chemistry & Detection Channel & $\begin{array}{c}\text { PCR Efficiency } \\
\text { (\%) }\end{array}$ \\
\hline amyloid & TaqMan probe & FAM & 96 \\
amyloid & TaqMan probe, BOXTO & FAM & 96 \\
amyloid & BOXTO & JOE & 98 \\
amyloid & TaqMan probe, BOXTO & JOE & 100 \\
gusA & LNA probe & FAM & 94 \\
gusA & LNA probe, BOXTO & FAM & 93 \\
gusA & BOXTO & JOE & 103 \\
gusA & LNA probe, BOXTO & JOE & 100 \\
Nanog & LNA probe, BOXTO & FAM/JOE & - \\
LNA, locked nucleic acid. & & \\
\hline
\end{tabular}

8.3 , and $50 \mathrm{mM} \mathrm{KCl}, 3 \mathrm{mM} \mathrm{MgCl}$, $0.3 \mathrm{mM}$ of each dNTP, $0.4 \mu \mathrm{M}$ of each primer, $1 \mathrm{U}$ JumpStart ${ }^{\mathrm{TM}}$ polymerase (Sigma-Aldrich), $0.1 \mu \mathrm{M}$ LNA probe, and $0.5 \mu \mathrm{M}$ BOXTO. Samples were run as triplicates (gusA and amyloid) and duplicates (nanog) in $20-\mu \mathrm{L}$ reactions using the Rotor-Gene 3000 (Corbett Research, Sydney, Australia). Cycting conditions for all systems were preincubation at $95^{\circ} \mathrm{C}$ for $3 \mathrm{~min}$, followed by 40 cycles of $95^{\circ} \mathrm{C}$ for $15 \mathrm{~s}$ and $60^{\circ} \mathrm{C}$ for $60 \mathrm{~s}$. Fluorescence was measured after each $60^{\circ} \mathrm{C}$ step on both the FAM channel (excitation wavelength 470 $\mathrm{nm}$, detection wavelength $510 \mathrm{~nm}$ ) and the JOE channel (excitation wavelength $530 \mathrm{~nm}$, detection wavelength $555 \mathrm{~nm}$ ). Melting curves were recorded after the run by stepwise temperature increase $\left(1{ }^{\circ} \mathrm{C} / 5 \mathrm{~s}\right)$ from $65^{\circ}$ to $95^{\circ} \mathrm{C}$, measuring the fluorescence on the JOE channel. Formation of expected PCR products was verified by agarose gel electrophoresis.

\section{RESULTS AND DISCUSSION}

We have tested the performance of three probe-based real-time PCR assays in the presence of the DNA binding dye BOXTO as an internal control for nonspecific product formation by melting curve analysis. One assay was based on a TaqMan probe, and the other two were based on LNA probes. The probes were labeled with FAM, and their fluorescence was measured on the instrument FAM channel, while BOXTO fluorescence was measured using the JOE channel. BOXTO was found to have negligible fluorescence in the FAM channel of the Rotor-Gene 3000 , thus being fully compatible with FAM-labeled probes.

Dilution series were performed for both amyloid and gusA assays, using probe, BOXTO, and a mixture of probe and BOXTO as detection chemistry reporters. For the reactions run with both probe and BOXTO as reporters, standard curves were constructed from both the FAM channel (probe) and JOE channel (BOXTO) signals. The standard curves are shown in Figure 1, $\mathrm{A}$ and $\mathrm{B}$ (data from reactions containing only BOXTO are not shown). The PCR efficiencies calculated from the standard curves based on the FAM signal were the same in the absence and presence of BOXTO, demonstrating that the presence of BOXTO does not inhibit the PCR (Table 1). The PCR efficiency was $96 \%$ for the amyloid assay and about $93 \%$ for the gusA assay. The PCR efficiencies calculated from standard curves based on the BOXTO signal were independent of the presence of probe (Table 1). The probe cycle threshold $\left(\mathrm{C}_{\mathrm{T}}\right)$ values were, however, somewhat different in the presence of BOXTO. With the TaqMan probe (the amyloid assay), the probe signal appeared about half a cycle earlier when BOXTO was present. The opposite was found with the LNA probe (the gusA assay). The reason for this shift of $C_{T}$ values is unclear. $A$ possibility is that the probes and bound BOXTO interact spectrally, resulting in small enhancement and small quenching, respectively, of the probe signal. In any case, the shifts do not affect quantification, since the slopes of the standard curves are the same in the presence and absence of BOXTO, and any differences in $\mathrm{C}_{\mathrm{T}}$ values between samples remain the same.

To demonstrate the advantage of combining a sequence-specific probe with a nonspecific dye in the same reaction, we tested the approach with amyloid and gusA assays that were not fully optimized and experienced primer-dimer formation. This was done by using regular Taq DNA polymerase instead of hot start Taq DNA polymerase. Standard curves based on the BOXTO signal level off at low DNA concentration, revealing formation of side products (dashed lines in Figure 1, A and B). From melting curve analysis of the BOXTO signal, the side product is seen to have a lower melting temperature than the targeted product, suggesting it represents primer-dimers. The amount of primer-dimers formed increases with decreasing DNA template concentration. The formation of primer-dimers also affects the reactions containing only probes as reporters. It consumes PCR reagents, whose concentrations eventually become so low that amplification efficiency is reduced, shifting the probe $\mathrm{C}_{\mathrm{T}}$ to higher values. This is evident in the serial dilution shown in Figure 1B. The sample indicated by "1" produces significant amounts of primer-dimers, and its probe $\mathrm{C}_{\mathrm{T}}$ is higher than would be expected without this interference, resulting in erroneous quantification. In the BOXTO channel, a lower $\mathrm{C}_{\mathrm{T}}$ is observed than expected in absence of primer-dimers, because BOXTO binds also to the primer-dimer products. The formation of primerdimers is evident from the melting curve analysis, where sample 1 has a large primer-dimer peak and a small product peak. It is noteworthy that the other two replicates of the sample do not produce significant amounts of primer-dimers (data not shown) and exhibit normal $C_{T}$ values. Primerdimer formation in real-time PCR is not very reproducible for the amyloid and gusA assays. In samples with even lower initial amounts of DNA, amplification of the targeted product is totally repressed, and no probe signal 
is detected (Figure 1, top of the graph). The advantage of using BOXTO in combination with sequence-specific probes is that more information about the reactions is obtained. In particular, the BOXTO signal reveals the formation of any primer-dimer products that may have interfered with the amplification of target product.

To further demonstrate the usefulness of combining probes and dyes in the same reaction, we studied the expression of nanog in human stem cell cultures at different degrees of differentiation (17). The nanog assay was highly optimized, generating very few primer-dimers when using hot start polymerase. However, even in this setup, the addition of BOXTO revealed more and interesting information that had not been observed using only probe. The expression of nanog over time is shown in Figure 1C. The melting curve analysis showed that nonspecific PCR products were formed in the samples with negligible nanog expression. The no template control was found to have products melting at lower temperature than the target PCR product (i.e., primer-dimers). Even though none of the positive samples showed signs of containing primer-dimers, samples with lower nanog concentrations could be affected by primer-dimers, and one should be aware that this data could be somewhat biased. The biological negative control had products with a higher melting temperature than the positive samples. This indicates that another product, not primer-dimers, has been formed (verified by gel electrophoresis, data not shown). Such a product formation could of course also affect the amplification of the correct product, especially if the latter is present at a low concentration.

In summary, we have shown that the nonspecific dsDNA binding dye BOXTO can be used in combination with sequence-specific FAM-based probes to monitor real-time PCR product formation. This combined system is advantageous to traditional probe- and dye-based assays by detecting both the formation of the targeted product and the interfering nonspecific products in a closed tube format. With this approach, samples exhibiting erroneous probe $\mathrm{C}_{\mathrm{T}}$ values due to competition with primer-dimer formation are readily identified. For critical measurements, this new approach offers most valuable quality assurance in real-time PCR-based analysis.

\section{ACKNOWLEDGMENTS}

This work was supported by the Swedish Research Council, the Carl Tryggers Foundation, and the EU FP6/2004/IST/NMP-2 program SMARTHEALTH (contract no. 016817). We thank Dr. Gunnar Westman for help with dye synthesis and for fruitful collaboration.

\section{COMPETING INTERESTS STATEMENT}

BOXTO is sold by TATAA Biocenter $A B$, Göteborg, Sweden. All authors have interest in TATAA Biocenter $A B$. N.Z. is employed by TATAA Biocenter $A B$.

\section{REFERENCES}

1.Stahlberg, A., P. Aman, B. Ridell, P. Mostad, and M. Kubista. 2003. Quantitative real-time PCR method for detection of Blymphocyte monoclonality by comparison of $\kappa$ and $\lambda$ immunoglobulin light chain expression. Clin. Chem. 49:51-59.

2.Stahlberg, A., N. Zoric, P. Aman, and M. Kubista. 2005. Quantitative real-time PCR for cancer detection: the lymphoma case. Expert Rev. Mol. Diagn. 5:221-230.

3. Richt, J.A., K.M. Lager, D.F. Clouser, E. Spackman, D.L. Suarez, and K.J. Yoon. 2004. Real-time reverse transcription-polymerase chain reaction assays for the detection and differentiation of North American swine influenza viruses. J. Vet. Diagn. Invest. 16:367-373.

4. Terry, C.F., D.J. Shanahan, L.D. Ballam, N. Harris, D.G. McDowell, and H.C. Parkes. 2002. Real-time detection of genetically modified soya using Lightcycler and ABI 7700 platforms with TaqMan, Scorpion, and SYBR Green I chemistries. J. AOAC Int. 85:938-944.

5. Elbing, K., A. Stahlberg, S. Hohmann, and L. Gustafsson. 2004. Transcriptional responses to glucose at different glycolytic rates in Saccharomyces cerevisiae. Eur. J. Biochem. 271:4855-4864.

6. Heid, C.A., J. Stevens, K.J. Livak, and P.M. Williams. 1996. Real time quantitative PCR. Genome Res. 6:986-994.

7. Tyagi, S. and F.R. Kramer. 1996. Molecular beacons: probes that fluoresce upon hybridization. Nat. Biotechnol. 14:303-308.

8. Caplin, B.E., R.P. Rasmussen, P.S. Bernard, and C.T. Wittwer. 1999. Lightcycler ${ }^{\mathrm{TM}}$ hybridization probes - the most direct way to monitor PCR amplification and mutation detection. Biochemica 1:5-8.

9. Svanvik, N., A. Stahlberg, U. Sehlstedt, R. Sjoback, and M. Kubista. 2000. Detection of PCR products in real time using light-up probes. Anal. Biochem. 287:179-182.

10. Wittwer, C.T., M.G. Herrmann, A.A. Moss, and R.P. Rasmussen. 1997. Continuous fluorescence monitoring of rapid cycle DNA amplification. BioTechniques 22:130-138.

11. Bengtsson, M., H.J. Karlsson, G. Westman, and M. Kubista. 2003. A new minor groove binding asymmetric cyanine reporter dye for real-time PCR. Nucleic Acids Res. 31:e45.

12. Ririe, K.M., R.P. Rasmussen, and C.T. Wittwer. 1997. Product differentiation by analysis of DNA melting curves during the polymerase chain reaction. Anal. Biochem. 245:154-160.

13.Sjöback, R., J. Nygren, and M. Kubista. 1995. Absorption and fluorescence properties of fluorescein. Spectrochim. Acta A 51:L7-L21.

14. Kubista, M. and N. Zoric. 2004. Real-time PCR platforms, p. 1126-1130. In J. Fuchs and M. Podda (Eds.), Encyclopedia of Medical Genomics and Proteomics. Dekker, New York.

15. Karlsson, H.J., M. Eriksson, E. Perzon, B. Akerman, P. Lincoln, and G. Westman. 2003. Groove-binding unsymmetrical cyanine dyes for staining of DNA: syntheses and characterization of the DNA-binding. Nucleic Acids Res. 31:6227-6234.

16. Zipper, H., H. Brunner, J. Bernhagen, and F. Vitzthum. 2004. Investigations on DNA intercalation and surface binding by SYBR Green I, its structure determination and methodological implications. Nucleic Acids Res. 32:e103.

17. Noaksson, K., N. Zoric, X. Zeng, M.S. Rao, J. Hyllner, H. Semb, M. Kubista, and P. Sartipy. 2005. Monitoring differentiation of human embryonic stem cells using real-time PCR. Stem Cells 23:1460-1467.

Received 4 July 2005; accepted 17 November 2005.

Address correspondence to Mikael Kubista, Department of Chemistry and Bioscience, Chalmers University of Technology, Lundbergslaboratoriet, Box 462, 40530 Gothenburg, Sweden. e-mail:Mikael.Kubista@tataa.com

\section{To purchase reprints}

of this article, contact

Reprints@BioTechniques.com 
